\title{
François Moureau, La plume et le plomb. Espaces de l'imprimé et du manuscrit au siècle des Lumières
}

\section{Franco Piva}

\section{(2) OpenEdition}

1 Journals

\section{Edizione digitale}

URL: http://journals.openedition.org/studifrancesi/8909

DOI: $10.4000 /$ studifrancesi.8909

ISSN: 2421-5856

\section{Editore}

Rosenberg \& Sellier

\section{Edizione cartacea}

Data di pubblicazione: 1 octobre 2008

Paginazione: 453-454

ISSN: 0039-2944

\section{Notizia bibliografica digitale}

Franco Piva, «François Moureau, La plume et le plomb. Espaces de l'imprimé et du manuscrit au siècle des Lumières», Studi Francesi [Online], 155 (LII | II) | 2008, online dal 30 novembre 2015, consultato il 12 janvier 2021. URL: http://journals.openedition.org/studifrancesi/8909 ; DOI: https://doi.org/10.4000/ studifrancesi.8909

Questo documento è stato generato automaticamente il 12 janvier 2021.

\section{(c)}

Studi Francesi è distribuita con Licenza Creative Commons Attribuzione - Non commerciale - Non opere derivate 4.0 Internazionale. 


\title{
François Moureau, La plume et le plomb. Espaces de l'imprimé et du manuscrit au siècle des Lumières
}

\author{
Franco Piva
}

\section{NOTIZIA}

FRANÇOIS MOUREAU, La plume et le plomb. Espaces de l'imprimé et du manuscrit au siècle des Lumières, Paris, PUPS, 2006, pp. 728.

1 Questo grosso volume di François Moureau può essere visto e letto come uno straordinario viaggio all'interno del mondo della comunicazione e dell'informazione in Francia nel Settecento. Il secolo dei Lumi è stato tradizionalmente visto come il secolo del libro. In quel periodo il libro conobbe infatti uno sviluppo straordinario, sia per le tecniche, sempre più raffinate, che furono messe a punto per la fabbricazione del libro, sia per il modo, sempre più capillare, con il quale il libro venne distribuito, sia per la concezione stessa, più utilitaristica, che del libro ci si fece in questo periodo, sia infine per il grande aumento dei lettori, facilitato tanto dalle migliori condizioni economiche del paese che dall'aumento del numero di coloro che sapevano leggere. È quanto ci ha lasciato intravvedere in questi ultimi decenni la «storia del libro», e che il volume di Fr. Moureau conferma ampiamente. Esso ci permette però anche di renderci conto che la situazione era assai più complessa di quanto finora si potesse pensare. I 37 capitoli che compongono il volume, frutto di un lavoro lungo una vita e basato su una conoscenza capillare degli archivi di mezza Europa, introducono in zone spesso poco note della storia del libro o forse sarebbe meglio dire dell'informazione, visto che esso va abbondantemente al di là del libro propriamente detto, rivelano aspetti poco conosciuti, fanno vedere situazioni insospettate, aprono prospettive alle quali, forse, non si era ancora pensato, o alle quali non era stata data l'importanza che invece meritavano. 
2 La produzione ed il commercio del libro erano, come è noto, soggetti ad una legislazione molto precisa, che passava attraverso la concessione di un privilegio il quale, a sua volta, richiedeva l'ottenimento di un nihil obstat da parte della censura, politica e religiosa; l'una e l'altra formalità prevedendo un controllo che, con il passare degli anni, si fece sempre più attento e preciso, a volte addirittura tatillon, ma proprio per questo difficilmente applicabile; tanto più che gli interessi politici e religiosi non potevano ignorare gli interessi economici che stavano dietro alla produzione del libro. Il libro era infatti, prima di tutto, il prodotto di un'industria, quella del libro appunto, che costituiva un settore importante e doveva essere difeso dall'agguerrita concorrenza proveniente dall'estero. Di qui ogni sorta di deroghe, di permissions tacites, di sotterfugi d'ogni genere cui tanto i librai che le autorità preposte al controllo ricorsero, in un gioco ambiguo di cache-cache e di connivenza, che Fr. Moureau mette ancor meglio in evidenza, sulla base dei documenti da lui scovati negli archivi parigini.

3 Nel Settecento l'informazione non passava tuttavia solo attraverso il libro, pur nelle forme assai più souples che esso assunse il quel periodo. Essa passava anche attraverso quello che con un termine generico, e almeno in parte improprio, possiamo chiamare il giornale. Dopo le ricerche coordinate da Jean Sgard è noto a tutti quelli che si interessano all'argomento quale importanza abbia assunto, nel Settecento, la stampa periodica: in crescita esponenziale lungo tutto il secolo, la stampa periodica, nelle forme diverse che essa assunse in questo periodo, ha rappresentato una delle fonti più importanti sia della diffusione dell'informazione che della formazione dell'opinione pubblica. Per questo le autorità cercarono fin dall'inizio di esercitare su di essa un controllo che, nella realtà, si è rivelato assai più difficile del previsto; tanto più che la diffusione dell'informazione, nel Settecento, avveniva non di rado, in forma manoscritta. Le «nouvelles à la main» svolsero, in questo ambito, un ruolo altrettanto importante delle «nouvelles imprimées». Lo sa benissimo Fr. Moureau che a questo settore dell'informazione ha dedicato molta parte della sua attività di ricerca, coordinando e pubblicamdo, alcuni anni orsono, un fondamentale Répertoire des nouvelles à la main, e che a questo aspetto dedica una parte importante anche del suo attuale volume; per mettere soprattutto in evidenza il gioco ambiguo, e per altro verso, estremamente interessante che si stabilì tra «nouvelles imprimées» e «nouvelles à la main» da un lato, tra «nouvellistes à la main» e autorità pubbliche da un altro; tra informazione più $\mathrm{o}$ meno controllata cioè $\mathrm{e}$ controinformazione più $\mathrm{o}$ meno ufficialmente accettata.

4 L'informazione diffusa «à la main» introduce ad un altro degli aspetti sui quali Fr. Moureau attira l'attenzione degli studiosi, vale a dire il ruolo che, nel Settecento, svolge ancora il manoscritto. Si sarebbe potuto pensare che, a fronte dell'enorme sviluppo conosciuto dal libro, il manoscritto sarebbe scomparso o sarebbe stato cantonné in un ambito molto ristretto. Le ricerche da lui svolte propongono una realtà assai più complessa. Non solo il manoscritto ha continuato ad esistere, ed in una percentuale assai più importante di quanto si sarebbe potuto supporre, ma con il libro stampato esso stabilì un rapporto assai articolato; nel quale l'aspetto economico svolgeva spesso un ruolo altrettanto importante di quello contenutistico. La diffusione manoscritta non riguardò, ad ogni modo, solo quelle opere che, per la loro natura sovversiva o scabrosa, non avrebbero mai potuto ottenere il visto della censura o il privilège dalle autorità (la cosiddetta «letteratura clandestina»). In fondo, questo ostacolo poteva essere superato abbastanza facilmente ricorrendo o all'edizione estera o alle stamperie più o meno 
clandestine, sempre disposte a stampare opere che avrebbero assicurato guadagni per i quali valeva ben la pena di rischiare condanne che, il più delle volte, finivano per ridursi a poca cosa. In realtà il rapporto tra «plomb» e "plume», per riprendere l'immagine proposta dal titolo del volume di Fr. Moureau, fu assai più complesso e obbedì a logiche che è opportuno avere ben presenti quando ci si accosta ad un secolo come il Settecento, sempre in bilico tra vecchio e nuovo, tra un rigore spesso più di facciata che realmente esercitato, e la volontà, anche da parte di chi quel rigore avrebbe dovuto applicare, di andare oltre, sotto la spinta di un nuovo cui pochi all'epoca furono, per un motivo o per un altro, insensibili.

Quelli ai quali abbiamo accennato non sono peraltro che alcuni, e forse neppure i più importanti o interessanti, dei numerosi aspetti toccati da Fr. Moureau nel suo libro, pieno di notizie curiose, di aperçus almeno in parte nuovi, di piste che meritano di essere ulteriormente percorse. Fr. Moureau non offre solo, come ha giustamente fatto osservare Robert Darnton nella «Préface», una preziosa «somme des connaissances acquises» nell'ambito della storia del libro e della comunicazione nella Francia dei Lumi. Il xviII secolo che il volume di Fr. Moureau propone è, per dirla ancora con R. Darnton, «un paysage varié, riche en contrastes, plein de surprises et libéré des frontières artificielles» dentro le quali una certa storiografia l'aveva, per motivi soprattutto ideologici, rinchiusa. Per cui il lettore, dopo aver chiuso questo grosso volume, non potrà non convenire che la Francia presentata da Fr. Moureau è «une destination qui vaut bien le voyage». 